\title{
PREPARACION DE CATALIZADORES BASADOS EN Ce-Pr PARA LA COMBUSTION DE n-HEXANO
}

\author{
Gino Picasso ${ }^{a^{*}}$, Pedro Navarro ${ }^{\mathrm{a}}$, César Zavala ${ }^{\mathrm{a}}$, Alcides López ${ }^{\mathrm{a}}$, Rosario Sun Kou ${ }^{\mathrm{b}}$
}

\begin{abstract}
RESUMEN
Catalizadores basados en óxidos mixtos de Ce-Pr, $\mathrm{Ce}_{\mathrm{x}} \mathrm{Pr}_{1-\mathrm{x}} \mathrm{O}_{2}$, fueron preparados por coprecipitación (con $\mathrm{x}$ de 0,1 a 0,9 ) para la combustión de n-hexano. Con fines de comparación, los correspondientes óxidos simples fueron también preparados. Todas las muestras fueron caracterizadas por sorción de $\mathrm{N}_{2}\left(\mathrm{~S}_{\mathrm{BET}}\right)$ y difracción de rayos $\mathrm{X}$ (XRD). El análisis textural de los catalizadores mostró la formación de isotermas tipo IV, con áreas superficiales entre 9,1 y $62,3 \mathrm{~m}^{2} / \mathrm{g}$, predominando la formación de mesoporos. Los patrones de difracción de rayos $\mathrm{X}$ de todas las muestras mostraron la presencia mayoritaria de la fase fluorita. La introducción de Pr en la composición del óxido mixto no alteró la estructura de tipo fluorita original. La evaluación catalítica se realizó en un reactor de lecho fijo, para una concentración inicial de n-hexano de 2000 ppm y con una velocidad espacial (WHSV) de $80 \mathrm{~h}^{-1}$. Las temperaturas de combustión total para los catalizadores mixtos estuvieron entre 356 y $437^{\circ} \mathrm{C}$. Con el óxido $\mathrm{Ce}_{0.9} \operatorname{Pr}_{0.1} \mathrm{O}_{2}$ se obtuvo la más alta velocidad específica (calculada por hora y gramo de catalizador, considerando la superficie específica), comparada con las correspondientes a la de los otros óxidos mixtos y óxidos simples puros como resultado de una mayor superficie específica obtenida.
\end{abstract}

Palabras clave: óxidos mixtos, Ce-Pr, eliminación de n-hexano

\section{PREPARATION OF CATALYST BASED ON Ce-Pr FOR COMBUSTION OF n-HEXANE}

\begin{abstract}
Catalysts based on Ce-Pr mixed oxides, $\mathrm{Ce}_{\mathrm{x}} \mathrm{Pr}_{1-\mathrm{x}} \mathrm{O}_{2}$, were prepared by coprecipitation (x ranged from 0,1 to 0,9 ) for combustion of $n$-hexane. For comparison purposes, the pure simple oxides were also prepared. Catalysts were characterized by sorption of $\mathrm{N}_{2}$ and X-ray diffraction (XRD). The analysis of adsorption isotherms of catalysts showed profiles of type $\mathrm{IV}$, with specific surfaces ranging from 9,1 to $62,3 \mathrm{~m}^{2} / \mathrm{g}$, with the prevalence of mesoporosity. The X-ray diffractogram patterns for pure and mixed oxide catalysts show the majority presence of stable fluorite phase. The introduction of Pr in mixed oxide composition maintains the crystal structure of fluorite-like type. The catalytic tests were carried out in a fixed bed reactor with a starting concentration of n-hexane of $2000 \mathrm{ppm}$ and weight hourly space velocity (WHSV) of $80 \mathrm{~h}^{-1}$. The total combustion temperatures for mixed oxides catalysts were in the range of 356 to $437^{\circ} \mathrm{C}$. The $\mathrm{Ce}_{0.9} \mathrm{Pr}_{0.1} \mathrm{O}_{2}$ oxide showed higher specific rate (evaluated per hour, gram of catalyst considering the specific surface) than the other

\footnotetext{
Laboratorio de Investigación de Fisicoquímica, Facultad de Ciencias, Universidad Nacional de Ingeniería, Av. Túpac Amaru 210, Rímac, Lima, Perú, *gpicasso@uni.edu.pe

b Departamento de Ciencias-Sección Química. Pontificia Universidad Católica del Perú. Avenida Universitaria 1801 - Lima 32.
} 
mixed oxides and pure simple oxides as a result of a higher value of surface area obtained.

Key words: mixed oxides, Ce-Pr, removal of n-hexane.

\section{INTRODUCCIÓN}

En septiembre 2005, la Comisión de las Comunidades Europeas, en el marco del programa "Clean Air for Europe" (CAFE), publicó su estrategia temática sobre la contaminación atmosférica. Esta estrategia establece los objetivos en la salud, en el ecosistema y en la reducción de emisiones para los principales contaminantes del aire. Para lograr estos objetivos, las emisiones de $\mathrm{SO}_{2}, \mathrm{NO}_{x}$, compuestos orgánicos volátiles (COV's), amoniaco y materia particulada $\mathrm{PM}_{2.5}$ deberán disminuir en $82 \%, 60 \%, 51 \%, 27 \%$ y $59 \%$, respectivamente, con relación a las emisiones registradas en el $2000^{1}$. La combustión catalítica es la técnica más eficaz de eliminación de COV's debido al empleo de menores temperaturas respecto a la incineración térmica, evitando la formación de óxidos de nitrógeno ${ }^{2}$. Los catalizadores empleados para la oxidación de COV's ${ }^{3}$ son óxidos soportados o no soportados conteniendo metales sólos o formulaciones complejas de los mismos. Como una alternativa a los metales nobles, los óxidos de metales de transición, en particular, los óxidos basados en ceria han resultado catalizadores prometedores para esta reacción ${ }^{4-6}$.

Los materiales basados en ceria (dióxido de cerio)son capaces de someterse a ciclos rédox $\mathrm{C} e^{4+} / \mathrm{Ce}^{3+}$ rápidos y reversibles, a temperaturas moderadas, debido a la alta movilidad de los iones metálicos desde la superficie del sólido hacia el seno del sistema (bulk) de reacción y viceversa ${ }^{7-9}$. En muchos casos, las propiedades rédox y la actividad química de la ceria pura pueden ser reforzadas mediante dopamiento con un segundo metal en la red cristalina del óxido $^{10-12}$. Entre los elementos reducibles, praseodimio, es particularmente adecuado para obtener disoluciones sólidas con cerio. En los óxidos mixtos, se puede formar estados de oxidación mixtos con ambos cationes $\mathrm{Pr}^{3+}$ y $\mathrm{Pr}^{4+}$. Además, en las soluciones sólidas Ce-Pr, ambos elementos pueden optar estados de oxidación $+3 \mathrm{y}+4$, y las vacancias aniónicas son más móviles en este sistema ${ }^{13-17}$. En trabajos previos se ha estudiado algunos catalizadores basados en Ce-Mn, Ce-Zr, Fe-Mn en la combustión de n-hexano, empleando catalizadores másicos y soportados; se determinó la existencia de una fuerte influencia de la estructura y composición del sistema mixto en la eficiencia de la reacción ${ }^{18-21}$. Este trabajo persigue estudiar la influencia de las propiedades estructurales del sistema mixto Ce-Pr en la combustión de n-hexano, un alcano muy difundido en nuestro país, especialmente como solvente en la industria química.

\section{PARTE EXPERIMENTAL}

Los materiales de partida en este trabajo fueron los siguientes:

- $\mathrm{Ce}\left(\mathrm{NO}_{3}\right)_{3} .6 \mathrm{H}_{2} \mathrm{O}_{(\mathrm{s})}$ Aldrich $99,99 \%$ de pureza.

- $\mathrm{Pr}\left(\mathrm{NO}_{3}\right)_{3} .6 \mathrm{H}_{2} \mathrm{O}_{(\mathrm{s})}$ Aldrich $99,99 \%$ de pureza.

- $\mathrm{NH}_{4} \mathrm{OH}_{(\text {conc })}$ Merck 99\% de pureza.

Los catalizadores fueron preparados en el Laboratorio de Investigación de Físicoquímica de la Facultad de Ciencias de la Universidad Nacional de Ingeniería. Los óxidos mixtos de ceriopraseodimio, $\mathrm{Ce}_{x} \mathrm{Pr}_{1-x} \mathrm{O}_{2} \quad(\mathrm{x}=0,1 ; 0,2 ; 0,35 ; 0,5 ; 0,65 ; 0,8$ y 0,9$)$ fueron preparados mediante el método de coprecipitación, usando $\left(\mathrm{NO}_{3}\right)_{3} .6 \mathrm{H}_{2} \mathrm{O}$ y $\mathrm{Pr}\left(\mathrm{NO}_{3}\right)_{3} \cdot 6 \mathrm{H}_{2} \mathrm{O}$ como precursores e hidróxido de amonio como agente precipitante. Las cantidades requeridas de cada precursor fueron disueltas en agua destilada y se mezclaron volúmenes en la proporción de los catalizadores deseados. Luego, se agregó hidróxido de amonio gota a gota $(1 \mathrm{~mL} / \mathrm{min})$ hasta 
que se completó la precipitación $(\mathrm{pH} \approx 9)$. El sólido formado (óxido/hidróxido) fue recuperado por filtración a vacío. El precipitado se secó en un horno a $110^{\circ} \mathrm{C}$ por $24 \mathrm{~h}$, luego se calcinó a $500^{\circ} \mathrm{C}$ por $1 \mathrm{~h}$ en aire estático. Finalmente, las muestras se tamizaron hasta obtener partículas de tamaño igual o menor a $150 \mu \mathrm{m}$.

Las reacciones químicas y procesos físicos que ocurren en cada etapa se muestran a continuación, donde M puede ser Ce o Pr:

Disolución:

$$
M\left(\mathrm{NO}_{3}\right)_{3} \cdot 6 \mathrm{H}_{2} \mathrm{O}_{(\mathrm{s})}+\mathrm{H}_{2} \mathrm{O}_{(l)} \rightarrow \mathrm{M}^{3+}{ }_{(a c)}+3 \mathrm{NO}_{3}^{-}{ }_{(a c)}+7 \mathrm{H}_{2} \mathrm{O}_{(l)}
$$

Precipitación:

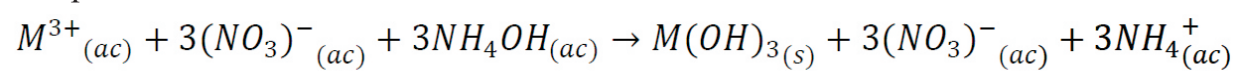

Secado:

Calcinación:

$$
\mathrm{H}_{2} \mathrm{O}_{(l)} \rightarrow \mathrm{H}_{2} \mathrm{O}_{(g)}
$$

$$
\mathrm{M}(\mathrm{OH})_{3(\mathrm{~s})}+0,25 \mathrm{O}_{2(g)} \rightarrow \mathrm{MO}_{2(\mathrm{~s})}+1,5 \mathrm{H}_{2} \mathrm{O}_{(\mathrm{g})}
$$

Como se observa, es en la etapa de precipitación donde se forma el hidróxido metálico, que después del proceso de calcinación se obtiene el correspondiente óxido metálico.

\section{RESULTADOS Y DISCUSIÓN}

\section{Caracterización por sorción de $\mathrm{N}_{2} \mathbf{Y}$ XRD.}

Las áreas superficiales de las muestras fueron determinadas por adsorción de $N_{2}$ a $-196^{\circ} \mathrm{C}$ usando un equipo Gemini VII automático modelo 2390t (Micromeritics). Previo al análisis, las muestras fueron desgasificadas por $2 \mathrm{~h} \mathrm{a} 250^{\circ} \mathrm{C}$ en un ambiente inerte de $\mathrm{He}$. Los resultados

\begin{tabular}{|c|c|c|c|c|}
\hline$n^{\circ}$ & Catalizador & $\mathrm{S}_{\mathrm{BET}}\left(\mathrm{m}^{2} / \mathrm{g}\right)$ & $\mathrm{d}_{\mathrm{BJH}}(\mathrm{nm})^{1}$ & $d(n m)^{2}$ \\
\hline 1 & $\mathrm{CeO}_{2}$ & 50,4 & 5,8 & 76,6 \\
\hline 2 & $C e_{0.9}{ } r_{0.1} O_{2}$ & 62,3 & 3,9 & 64,2 \\
\hline 3 & $\mathrm{Ce}_{0.8} \mathrm{Pr}_{0.2} \mathrm{O}_{2}$ & 60,6 & 6,7 & 77,2 \\
\hline 4 & $\mathrm{Ce}_{0.65} \mathrm{Pr}_{0.35} \mathrm{O}_{2}$ & 28,4 & 18,2 & 76,6 \\
\hline 5 & $\mathrm{Ce}_{0.5} \mathrm{Pr}_{0.5} \mathrm{O}_{2}$ & 53,4 & 19,8 & 63,7 \\
\hline 6 & $C e_{0.35} \mathrm{Pr}_{0.65} \mathrm{O}_{2}$ & 22,2 & 19,1 & 76,6 \\
\hline 7 & $\mathrm{Ce}_{0.2} \mathrm{Pr}_{0.8} \mathrm{O}_{2}$ & 48,9 & 27,6 & 76,6 \\
\hline 8 & $C e_{0.1} P_{0.9} O_{2}$ & 9,1 & 31,2 & 95,9 \\
\hline 9 & $\mathrm{PrO}_{2}$ & 37,4 & 20,3 & 89,3 \\
\hline
\end{tabular}
de la caracterización textural de los catalizadores frescos (sin uso) se muestran en la tabla 1.

Tabla 1. Superficies específicas y diámetro medio de poro (método BJH) y tamaño de cristalita de los catalizadores frescos ( $\sin$ uso).

${ }^{1}$ Calculado por el método Barrett-Joyner-Halenda, ${ }^{2}$ Calculado por la fórmula de Scherrer: $d(n m)=\frac{K \cdot \lambda}{\beta \cdot \cos \theta}$ donde $\mathrm{K}=0,1 ; \lambda=1,5418 \AA ; \beta$ : ancho del pico y $\theta$ : ángulo de difracción. 
Todos los catalizadores han presentado isotermas tipo IV que corresponden a sólidos micromesoporosos con formación preferente de mesoporosidad (figura 1). La presencia de pequeños ciclos de histéresis del tipo $\mathrm{H} 1$ (que se atribuye a sólidos con poros estrechos o cilíndricos), aparecen en las isotermas de los catalizadores mixtos $\mathrm{Ce}_{0.8} \mathrm{Pr}_{0.2} \mathrm{O}_{2}$, $\mathrm{Ce}_{0.65} \mathrm{Pr}_{0.35} \mathrm{O}_{2}$ y $\mathrm{Ce}_{0.5} \mathrm{Pr}_{0.5} \mathrm{O}_{2}$, talizador puro de $\mathrm{PrO}_{2}$, mientras que en la isoterma correspondiente al óxido mixto equimolar se observó un ciclo de histéresis del tipo $\mathrm{H} 3$, con formación preferente de poros laminares. Por otro lado, la isoterma del $\mathrm{Ce}_{0.9} \mathrm{Pr}_{0.1} \mathrm{O}_{2}$ presenta un comportamiento similar a la isoterma del $\mathrm{CeO}_{2}$.

La inserción de Pr en la estructura del produce un mayor aumento del área en el catalizador $C e_{0.9} P r_{0.1} \mathrm{O}_{2}$ de $50,4 \mathrm{a} 62,3 \mathrm{~m}^{2} / \mathrm{g}$ y una disminución en el diámetro de poro de 5,8 hasta $3,9 \mathrm{~nm}$, para luego aumentar paulatinamente con la presencia del Pr hasta 31,2nm (tabla 1).

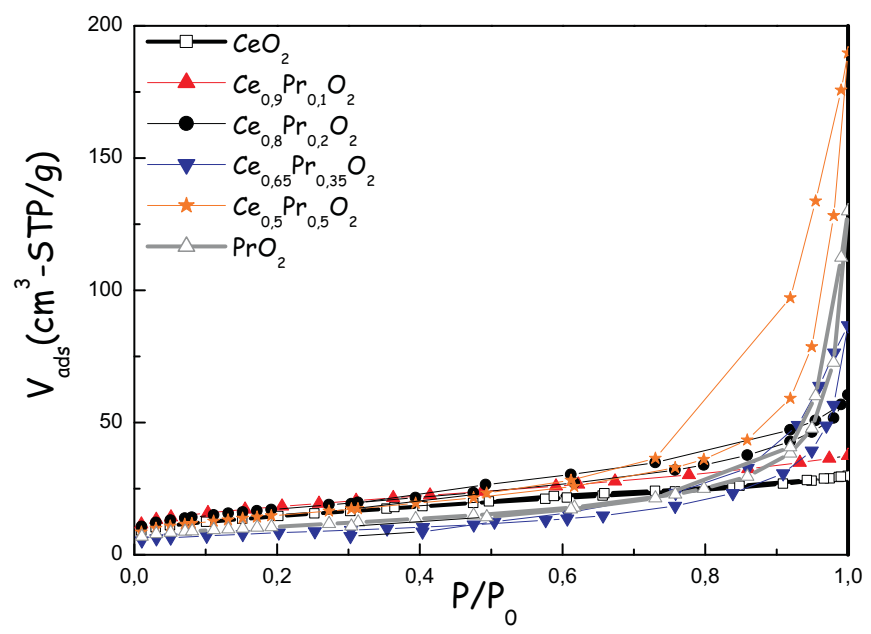

Figura 1. Evolución de las isotermas al aumentar la concentración de Pr en los catalizadores.

La figura 2 muestra los difractogramas de rayos X de los catalizadores frescos. La muestra de $\mathrm{CeO}_{2}$ presentó la estructura fluorita estable, típica de este material (JCPDS file: 34-0394). Los picos de difracción XRD de los catalizadores mixtos frescos aparecieron en la misma posición que los de $\mathrm{CeO}_{2}$; sin embargo, para el caso del $\mathrm{PrO}_{2}$ se observa un corrimiento de los picos hacia menores ángulos, que es consistente con la formación de dos posibles especies cristalinas: $\mathrm{PrO}_{1.83}$ o $\mathrm{Pr}_{6} \mathrm{O}_{11}$ (JCPDS file: 06-0329 y 42-1121, respectivamente) y los hombros, observados a menores ángulos, sugieren la presencia de la fase $\mathrm{PrO}_{2}$ (JCPDS file: 24-1006) ${ }^{13}$. Los difractogramas de las muestras mixtas mostraron tener la misma estructura, tipo fluorita, de la ceria pura con un ligero aumento del ancho de los picos a medida que aumenta el contenido de Pr. El tamaño de cristalita varió entre 63 y $96 \mathrm{~nm}$, con tendencia a aumentar a mayores contenidos de Pr; su magnitud guarda una correlación lineal aproximada con la magnitud de la superficie específica. 


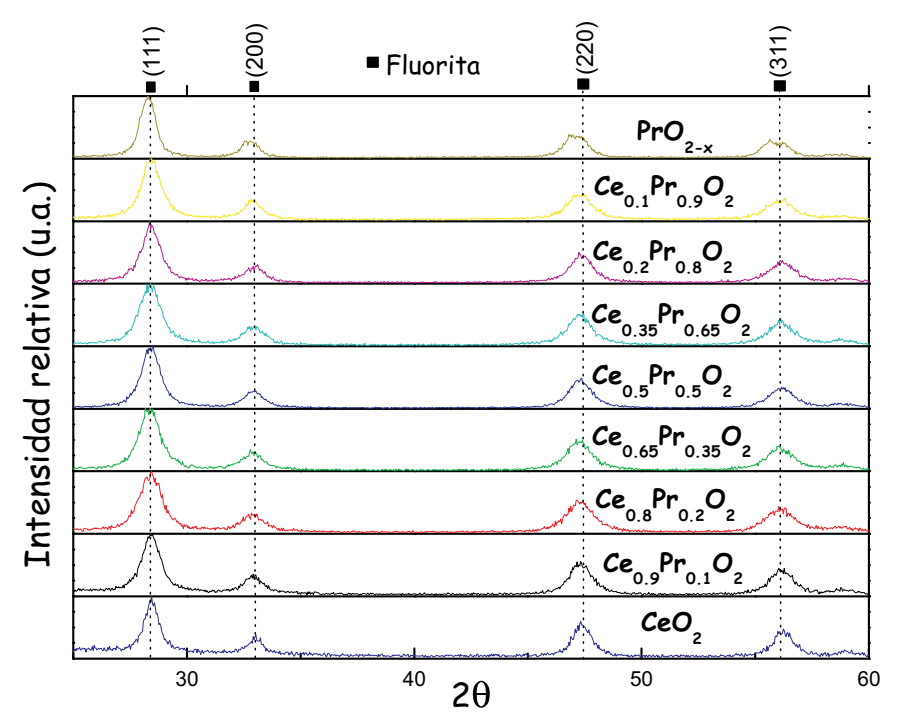

Figura 2. Difractogramas XRD de los catalizadores de Ce-Pr preparados.

\section{Evaluación de la actividad catalítica.}

Los catalizadores puros y mixtos basados en Ce-Pr, fueron ensayados en el reactor de lecho fijo que consiste en un tubo de cuarzo de $60 \mathrm{~cm}$ de largo y $0,9 \mathrm{~cm}$ de diámetro interno con una frita fina de cuarzo dentro de un horno hexagonal, similar al descrito en trabajos anteriores ${ }^{18-21}$. En el interior del reactor, en contacto con el lecho fijo se colocó un termopar tipo K. El lecho fijo consistió en una mezcla $100 \mathrm{mg}$ de catalizador con $200 \mathrm{mg}$ de cuarzo pulverizado, ambos previamente pasados a través de un tamiz de $150 \mu \mathrm{m}$. Como flujo de alimentación al reactor se utilizó una corriente de aire saturada con $n$-hexano a $0^{\circ} \mathrm{C}$, esto se consiguió empleando una batería de 3 saturadores, para una concentración inicial del alcano de 2000 ppm y velocidad espacial respecto al peso del lecho de $80 \mathrm{~h}^{-1}$. Para homogenizar el reactor con la corriente de alimentación se dejó pasar el aire saturado de n-hexano a $0^{\circ} \mathrm{C}$; durante aproximadamente 11 horas; esto se realizó un día antes del ensayo. Luego de la homogenización, la corriente de aire saturada con hexano se analizó por cromatografía de gases para verificar que la concentración inicial de hexano alimentado sea de $2000 \mathrm{ppm}$. Luego se dejó el horno a $100^{\circ} \mathrm{C}$ durante 30min para poder estabilizar el sistema. Después se realizaron los ensayos en el cromatógrafo por duplicado, obteniendo así los cromatogramas para la combustión catalítica de n-hexano a la temperatura inicial de $100^{\circ} \mathrm{C}$. Para determinar el efecto de la temperatura se realizó, de manera análoga a la descrita, una serie de ensayos en el intervalo de $100^{\circ} \mathrm{Ca} 450^{\circ} \mathrm{C}$, con intervalos de $30{ }^{\circ} \mathrm{C}$ hasta obtener una conversión del $95 \%$. Después de terminar los análisis a distintas temperaturas, se verificó la concentración inicial de n-hexano.

La conversión catalítica de las muestras mixtas se inició aproximadamente a $250^{\circ} \mathrm{C}$ y alcanzó la conversión de $95 \%$ a temperaturas menores de $440^{\circ} \mathrm{C}$ (figura 3 y tabla 2). La inserción de cierta cantidad de praseodimio en la red cristalina de la ceria involucró un efecto positivo en la 
actividad del óxido mixto resultante. Particularmente, con el catalizador mixto $\mathrm{Ce}_{0.9} \mathrm{Pr}_{0.1} \mathrm{O}_{2}$ se logró una mayor conversión que las obtenidas con los correspondientes óxidos simples puros y los otros óxidos mixtos, debido probablemente a una mayor superficie específica (tabla 2).

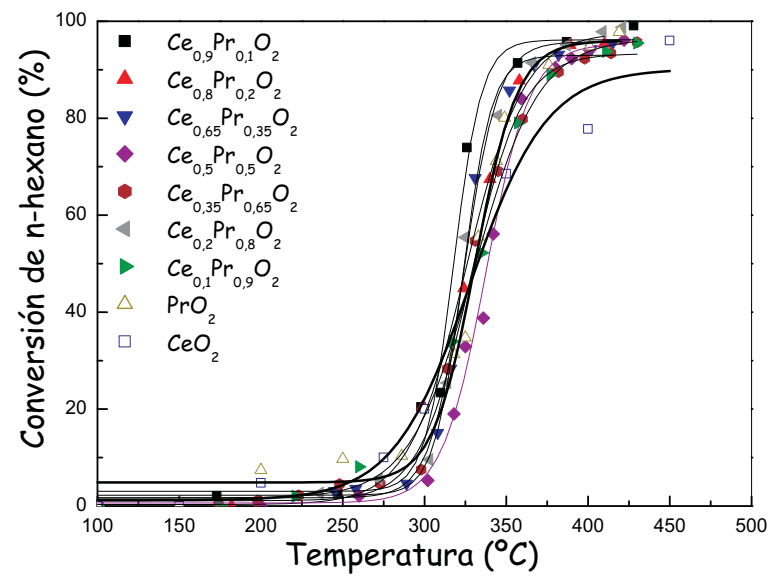

Figura 3. Curvas de ignición de los catalizadores basados en óxidos simples $\mathrm{CeO}_{2}, \mathrm{PrO}_{2} \mathrm{y}$ en óxidos mixtos $\mathrm{Ce}_{\mathrm{x}} \mathrm{Pr}_{1-\mathrm{x}} \mathrm{O}_{2}(\mathrm{x}=0,1$ a 0,9$)$ obtenidas en la combustión de n-hexano.

Tabla 2. Temperatura de combustión total $\left(\mathrm{T}_{95 \%}\right)$ y cantidad de milimoles de $\mathrm{n}$-hexano convertido a $400^{\circ} \mathrm{C}$ de los catalizadores Ce-Pr en la combustión de n-hexano.

\begin{tabular}{ccc}
\hline Catalizador & $\mathbf{T}_{\mathbf{9 5 \%}}\left({ }^{\circ} \boldsymbol{C}\right)$ & $\begin{array}{c}\text { mmol } \\
\text { n-hexano }\end{array}$ \\
\hline $\mathrm{CeO}_{2}$ & 450,0 & 19,8 \\
$\operatorname{Pr}_{2} \mathrm{O}_{2}$ & 389,5 & 19,1 \\
$\mathrm{Ce}_{0.1} \operatorname{Pr}_{0.9} \mathrm{O}_{2}$ & 437,0 & 4,6 \\
$\mathrm{Ce}_{0.2} \operatorname{Pr}_{0.8} \mathrm{O}_{2}$ & 374,1 & 25,1 \\
$\mathrm{Ce}_{0.35} \operatorname{Pr}_{0.65} \mathrm{O}_{2}$ & 422,8 & 10,9 \\
$\mathrm{Ce}_{0.5} \operatorname{Pr}_{0.5} \mathrm{O}_{2}$ & 402,8 & 27,1 \\
$\mathrm{Ce}_{0.65} \operatorname{Pr}_{0.35} \mathrm{O}_{2}$ & 407,6 & 14,2 \\
$\mathrm{Ce}_{0.8} \operatorname{Pr}_{0.2} \mathrm{O}_{2}$ & $\mathbf{3 8 8 , 2}$ & $\mathbf{3 1 , 2}$ \\
$\mathrm{Ce}_{0.9} \operatorname{Pr}_{0.1} \mathrm{O}_{2}$ & $\mathbf{3 5 6 , 2}$ & $\mathbf{3 2 , 2}$ \\
\hline
\end{tabular}

La medición de la actividad catalítica se realizó evaluando la velocidad específica en milimoles de n-hexano convertidos por hora, gramo de catalizador, considerando la superficie específica. Las ecuaciones de aplicación en detalle se presentaron en trabajo previo $^{22}$. Como se observa de la figura 4, las diferencias en actividad entre los catalizadores mixtos y simples son más pronunciadas, siendo las muestras con mayor contenido de $\mathrm{Ce}$ $\left(\mathrm{Ce}_{0.9} \operatorname{Pr}_{0.1} \mathrm{O}_{2}\right.$ y $\left.\mathrm{Ce}_{0.8} \operatorname{Pr}_{0.2} \mathrm{O}_{2}\right)$ las que presentaron mayor cantidad de alcano convertido que corresponden a una mayor superficie específica obtenida (tabla 1). El aumento de una pequeña 
cantidad de Pr en la estructura del óxido mixto permitió un aumento pronunciado de 19,8 mmol de n-hexano convertido en $\mathrm{CeO}_{2}$ hasta 32,2 con el catalizador $\mathrm{Ce}_{0.9} \operatorname{Pr}_{0.1} \mathrm{O}_{2}$ y 31,2 con el $\mathrm{Ce}_{0.8} \operatorname{Pr}_{0.2} \mathrm{O}_{2}$. Estos resultados indicarían que el reemplazo de un átomo de mayor tamaño como el Pr en la estructura de la fluorita parece mejorar el grado de reducibilidad de los catalizadores y el enriquecimiento superficial del Pr determinado por análisis XPS (resultados no mostrados), como sugieren otros autores ${ }^{13}$.

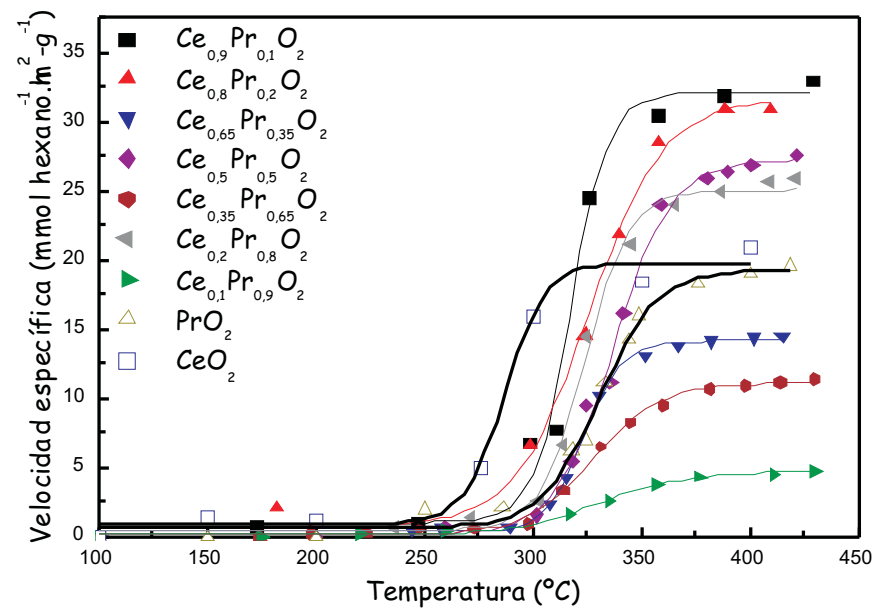

Figura 4. Curvas de velocidad específica de los catalizadores simples $\mathrm{CeO}_{2}, \mathrm{PrO}_{2} \mathrm{y}$ de los mixtos $\mathrm{Ce}_{\mathrm{x}} \mathrm{Pr}_{1-\mathrm{x}} \mathrm{O}_{2}$ (x de 0,1 a 0,9 ) en combustión de hexano.

\section{CONCLUSIONES}

- Se preparó catalizadores mixtos de $\mathrm{Ce}_{x} \mathrm{Pr}_{1-x} \mathrm{O}_{2}$, con las siguientes composiciones $\mathrm{x}=0$, 0,$1 ; 0,2 ; 0,35 ; 0,5 ; 0,65 ; 0,8 ; 0,9$ y 1,0

- Se caracterizó los catalizadores mediante las técnicas de sorción de $N_{2}$ y XR, mostrando texturas preferentemente mesoporosas, con superficies específicas entre 9,1 y $62,3 \mathrm{~m}^{2} / \mathrm{g}$. Los difractogramas mostraron que la introducción de Pr en el catalizador mantuvo la estructura tipo fluorita estable.

- Conel catalizador $\mathrm{Ce}_{0.9} \mathrm{Pr}_{0.1} \mathrm{O}_{2}$ se logró una mayor actividad comparada con las muestras simples debido probablemente a una mayor superficie específica $\left(\mathrm{S}_{\mathrm{BET}}\right)$ y mejor posicionamiento del Pr en el óxido mixto.

\section{AGRADECIMIENTO}

Los autores agradecen al Instituto General de Investigación (IGI) de la Universidad Nacional de Ingeniería por el apoyo económico concedido para la realización de este trabajo durante el año 2014. 


\section{REFERENCIAS}

1. Thematic Strategy on Air Pollution Communication from the Commission to the Council and The European Parliament, Commission of the European Communities, COM (2005) 446 final, Brussels, 2005, p 2-5.

2. Papaefthimiou P., Ionnides T., VerykiosX. E., Combustion of non-halogenated volatile organic compounds over group VIII metal catalysts, Appl. Catal. B 1997; 13: 175-184.

3. Fierro J.L.G.(Editor), Metal Oxides Chemistry and Applications, CRC Press, U.S.A. 2006, p. 215-236.

4. Perez A., Molina R., Moreno S., Enhanced VOC oxidation over Ce/CoMgAl mixed oxides using a reconstruction method with EDTA, Appl. Catal, A 2014; 477: 109-116.

5. Azalim S., Brahmi R., Agunaou M., Beaurain A., Giraudon J.M., Lamonier J.F., Washcoating of cordierite honeycomb with $\mathrm{Ce}-\mathrm{Zr}-\mathrm{Mn}$ mixed oxides for VOC catalytic oxidation, Chem. Eng. J. 2013; 223: 536-546.

6. De Rivas B., Sampedro C., García-Real M., López-Fonseca R., Gutiérrez-Ortiz J.I., Promoted activity of sulphated $\mathrm{Ce} / \mathrm{Zr}$ mixed oxides for chlorinated VOC oxidative abatement, Applied Catalysis B 2013; 129: 225-235.

7. Vicario M., Llorca J., Boaro M., De Leitenburg C., Trovarelli A., Redox behavior of gold supported on ceria and ceria-zirconia based catalysts, J. Rare Earth 2009; 27 (2): 196203.

8. Aneggi E., Boaro M., De Leitenburg C., Dolcetti G., Trovarelli A., Insights into the dynamics of oxygen storage/release phenomena in model ceria-zirconia catalysts as inferred from transient studies using $\mathrm{H}_{2}, \mathrm{CO}$ and soot as reductants, Catal. Today 2006; 112: 94-98.

9. Boaro M., Giordano F., Recchia S., Dal Santo V., Giona M., Trovarelli A., On the mechanism of fast oxygen storage and release in ceria-zirconia model catalysts, Appl. Catal. B 2004; 52: 225-237.

10. Konsolakis M., Carabineiro S.A.C., Tavares P.B., Figueiredo J.L., Redox properties and VOC oxidation activity of $\mathrm{Cu}$ catalysts supported on $\mathrm{Ce}_{1-\mathrm{x}} \mathrm{Sm}_{\mathrm{x}} \mathrm{O}_{\delta}$ mixed oxides, $J$. Hazard. Mat. 2013;261:512-521.

11. Solsona B., Garcia T., Aylón E., Dejoz A.M., Vázquez I., Agouram S., Davies T.E., Taylor S., Promoting the activity and selectivity of high surface area Ni-Ce-O mixed oxides by gold deposition for VOC catalytic combustion, Chem. Eng. J. 2011; 175: 271278.

12. Barakat T., Idakiev V., Cousin R., Shao G.S., Yuan Z.Y., Tabakova T., Siffert S., Total oxidation of toluene over noble metal based $\mathrm{Ce}, \mathrm{Fe}$ and Ni doped titanium oxides, Appl. Catal. B 2014; 146: 138-146.

13. De Rivas b., Guillén-Hurtado n., López-Fonseca R., Coloma-Pascual F., García-García A., Gutiérrez-Ortiz J.I., Bueno-López A., Activity, selectivity and stability of praseodymium-doped $\mathrm{CeO}_{2}$ for chlorinated VOCs catalytic combustion, Appl. Catal. B 2012; 121-122: 162-170.

14. Cabral A.C., Cavalcante L.S., Deus R.C., Longo E., Simões A.Z., Moura F., Photoluminescence properties of praseodymium doped cerium oxide nanocrystals, Ceram. Int. 2014; 40 (3): 4445-4453.

15. Biswas M., Bandyopadhyay S., Nano-crystalline $\operatorname{Pr}^{3+}$-ceria powder by ureaformaldehyde gel combustion route, Advanced Powder Technology 2014; 25(2): 536542. 
16. Kaddouri A., Béguin B., Methane steam reforming in the absence and presence of $\mathrm{H}_{2} \mathrm{~S}$ over $\mathrm{Ce}_{0.8} \mathrm{Pr}_{0.2} \mathrm{O}_{2-\delta} ; \mathrm{Ce}_{0.85} \mathrm{Sm}_{0.15} \mathrm{O}_{2-\delta}$ and $\mathrm{Ce}_{0.9} \mathrm{Gd}_{0.1} \mathrm{O}_{2-\delta}$ SOFCs anode materials, Catal. Commun. 2014; 46:22-27.

17. Bueno-LópezA., Diesel soot combustión ceria catalysts, Appl. Catal. B 2014; 146: 1-11.

18. Picasso G., Sun Kou M.R., Zavala C., Cruz R., López A., Preparación de catalizadores basados en óxidos mixtos de Ce/Mn por coprecipitación para la combustión de nhexano, Revista de la Sociedad Química del Perú 2013; 79(4):367-380.

19. Picasso, G., Sun Kou, M.R., Salazar I., Síntesis de catalizadores nanoestructurados basados en óxido de Mn para la eliminación de n-hexano, Revista de la Sociedad Química del Perú 2011; 77 (1): 11-26.

20. Picasso G., Sun Kou M.R., Gómez G., Hermoza E., López A., Pina M.P., Herguido J., Catalizadores nanoestructurados basados en óxidos de Fe para la combustión de nhexano", Revista de la Sociedad Química del Perú 2009; 75 (2): 163-176.

21. Picasso G., Gutiérrez M., Pina M.P., Herguido J., Preparation and characterization of Ce$\mathrm{Zr}$ and Ce-Mn based oxides for n-hexane combustion: Application to catalytic membrane reactors, Chem.Eng. J. 2007; 126: 119-130.

22. Picasso G., Belleza F., Zavala C., López A., Sun Kou M. R., Preparación de catalizadores basados en $\mathrm{Cu}-\mathrm{Mn}$ para la combustión de n-hexano, Revista de la Sociedad Química del Perú 2014, en imprenta. 